\title{
High Pressure Crystallization of Ultra-High Molecular Weight Polyethylene
}

\author{
Munehisa YASUNIWA and Chitoshi NAKAFUKU* \\ Faculty of Science, Fukuoka University, Jonan-ku, Fukuoka 814-01, Japan \\ *Faculty of Education, Kochi University, Kochi 780, Japan
}

(Received July 5, 1986)

\begin{abstract}
The morphology of pressure-crystallized ultra-high molecular weight $\left(2.5 \times 10^{6}\right)$ polyethylene samples was studied by scanning electron microscopy. The DSC and small angle Xray analyses were also performed. High pressure crystallization (HPC) (at a cooling rate of $2 \mathrm{~K} \mathrm{~min}^{-1}$ from the melt at $590 \mathrm{MPa}$ ), of an as-polymerized powder sample gives a fibrous band in morphology. The fibrous band is formed by the parallel arrangement of long fibrils whose diameters are less than about $500 \AA$. The width of the fibrous band is about $0.2 \mu \mathrm{m}$. The HPC of a bulk sample which was kneaded in the molten state at atmospheric pressure gives three types in its morphology: isolated fibril, textile structure and band structure. The textile structure is the network superstructure of the fibrils, and the band structure is also formed by the parallel arrangement of the fibrils. The parallel arrangement of the fibrils induced by the pressure in the molten state occurs prior to the growth of extended-chain crystals (ECC). The heat of fusion of the pressure-crystallized powder sample is much lower than that of an ECC sample of medium molecular weight PE. Though the lamellar thickness of powder and bulk samples increases by HPC, that of the pressurecrystallized bulk is not so large, and its distribution is wide.
\end{abstract}

KEY WORDS Polyethylene / High Pressure / Crystallization / Ultra High Molecular Weight / Extended-Chain Crystal / Fibril / Entanglement / Electron Microscopy / Morphology /

The formation of so-called "extended-chain crystal" (ECC) of polyethylene (PE) on the crystallization from the melt under pressure was first revealed by Wunderlich et al. ${ }^{1,2}$ Since then high pressure crystallization (HPC) of PE has been studied by many investigators. Pressure-crystallized PEs have been made under various crystallization conditions, and the characteristics of these samples have been clarified by various methods. The HPC of PE has been reviewed by Wunderlich, ${ }^{3-5}$ Bassett, ${ }^{6,7}$ Bhateja and Pae,${ }^{8}$ and Leute and Dollhopf. ${ }^{9}$

The investigation on the HPC of PE has been focused on medium and high molecular weight PE (MMW-, HMW-PE) ranging from $10^{3}$ to $10^{5}$. Recently the differential thermal analysis (DTA) under pressure for a series of
PE from low $\left(2 \times 10^{3}\right)$ to ultra-high molecular weight (UHMW) $\left(2.5 \times 10^{6}\right)$ has been reported. ${ }^{10}$ It has been presented that the DTA curves of melting under high pressure for pressure-crystallized samples of as-polymerized UHMW-PE (powder) show sharp endothermic peaks, and the peak temperatures are higher than those of MMW-PE. This result indicates that a rapid formation of ECC occurs in UHMW-PE as in the case of MMW-PE. ${ }^{11}$ However, it is easily supposed that the chain length of UHMW-PE is too long for the rapid formation of ECC taking into accounts the band width. A fibrillar morphology appears in the as-polymerized UHMW-PE in the scanning electron microscope (SEM) observation. The formation of a closely spaced band structure from the fibrillar 
structure by the HPC is supposed not to be easy in the case of UHMW-PE. It is interesting to investigate the formation mechanism of ECC and the band structure of UHMW-PE under high pressure.

The HPC of UHMW-PE has been investigated by Hoehn et al. ${ }^{12,13}$ They carried out electron microscopy on the pressure-crystallized PE with different molecular weights and concluded that the dominant feature in the morphology of UHMW-PE was a network superstructure consisting of or containing irregular and disordered extended chain lamellae.

For the pressure-crystallized UHMW-PE bulk sample which was prepared by kneading powder sample in the molten state at atmospheric pressure, the melting DTA peaks were smaller and broader, and the peak temperatures are lower than those of the pressurecrystallized powder sample. ${ }^{10}$ It has been assumed that the difference of the melting behavior between the powder and bulk samples is related to the number of entanglements of a long chain molecule in these sample. Such a structural difference in the molecular level should be reflected in the morphology of these samples.

The primary object of this paper is to clarify the morphology of pressure-crystaliized UHMW-PE and to examine the formation mechanism of the band structure. The secondary point is to elucidate the microscopic structural difference between the pressurecrystallized powder and bulk samples.

\section{EXPERIMENTAL}

Unfractionated UHMW-PE was supplied by Showa Denko Co. The viscosity-averaged molecular weight is $2.5 \times 10^{6}$. Two types of samples, powder and bulk, were used in this experiment. The powder sample was an aspolymerized UHMW-PE. The bulk sample was a melt-crystallized (kneaded) one. The detailed procedure for the preparation of the bulk sample is as follows. A powder sample was melted at about $170^{\circ} \mathrm{C}$ on a hot plate, and then kneaded by a spatula. As the melt viscosity was high, a filmy sample could be made by pressing and spreading the melt. After folding the filmy sample several times over, the sample was kneaded, pressed and spread again. This procedure was performed several times within about five minutes. Finally the bulk sample was obtained by the rapid cooling of the melted sample to room temperature.

HPC of the sample was carried out by the use of the high pressure DTA apparatus described elsewhere. ${ }^{14}$ The heating and cooling rates were precisely controlled by an automatic temperature controller. The hydrostatic pressure was measured within $\pm 1 \mathrm{MPa}$ by a Bourdon gauge (Heise) connected to the high pressure vessel.

A powder sample was prepared in the form of a rod (ca. $1.2 \mathrm{~mm}$ in diameter and $10 \mathrm{~mm}$ in length) by compressing the original powder sample in a piston cylinder type cell at room temperature. A bulk sample was prepared in the same shape as the rod powder sample by shaving the original bulk sample with a razor blade. In HPC, the samples were coated by epoxy resin to avoid the effect of the pressuretransmitting fluid (silicone oil). The crystallization procedure was as follows. The sample was heated up to $295^{\circ} \mathrm{C}$ at $5 \mathrm{~K} \mathrm{~min}^{-1}$ and held at $295^{\circ} \mathrm{C}$ for two minutes at $590 \mathrm{MPa}$. It was then cooled at $2 \mathrm{Kmin}^{-1}$ under the same pressure. Melting peak temperatures of the high pressure phase (hexagonal phase) in high pressure DTA for the powder and bulk samples at $590 \mathrm{MPa}$ are 267 and $255^{\circ} \mathrm{C}$, which are calculated from the phase diagrams of these samples, respectively. ${ }^{10}$ In this procedure, the powder and bulk samples were held in the molten state for about 20 and 28 minutes, respectively.

Specimens for the electron microscopy were prepared by fracturing the PE rods in liquid nitrogen. Rod samples obtained by HPC were brittle-fractured. The fracture surface was 
coated with $\mathrm{Au}$ and examined with a Hitachi S-430 SEM at magnification up to $30000 \times$. High magnification electron micrographs (up to $50000 \times$ ) were taken by Hitachi, Ltd. with a Hitachi S-800 field emission type SEM which is guaranteed a resolution of $20 \AA$

The small-angle $\mathrm{X}$-ray scattering (SAXS) profiles were obtained by using a Rigaku Denki small-angle X-ray diffractometer. Nickel filtered $\mathrm{Cu} K_{\alpha}$ radiation was used as an incident X-ray beam. DSC measurements were made with a Rigaku Denki low temperature thermal analyzer. Indium was used as calibration standard.

\section{RESULTS AND DISCUSSION}

Scanning electron micrographs of a fracture surface for pressure-crystallized powder samples of UHMW-PE are shown in Figures 1(a), (b), and (c). The macrostructure at low magnification of the sample is shown in Figure 1(a). The sample is composed of an aggregate of many particles of diameter about $10 \mu \mathrm{m}$ or above and is very porous.

Figures 1(b) and (c) show microstructures of the sample. As shown in these figures a band like structure develops in the sample, but the structure is strikingly different from the band structure of the pressure-crystallized

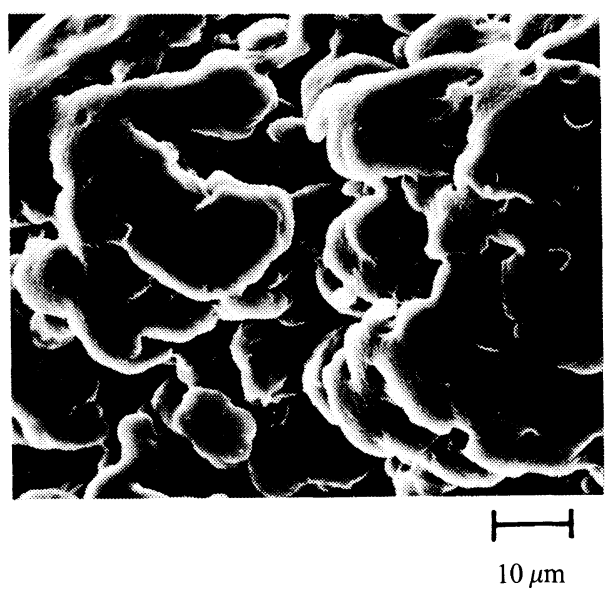

(a)
MMW-PE samples which have been reported by many authors. That is, instead of the growth of closely spaced extended-chain lamellae with striation, a fibrous band which consists of many long fibrils of diameter less than about $500 \AA$ grows sparsely. Arrows in

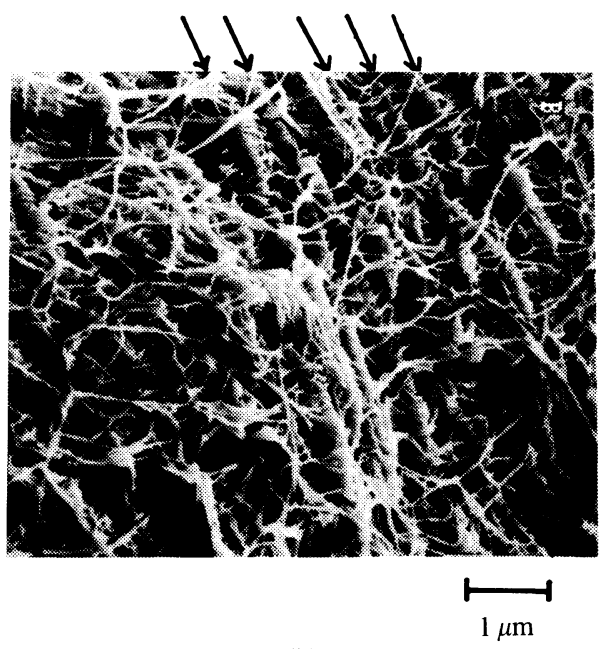

(b)

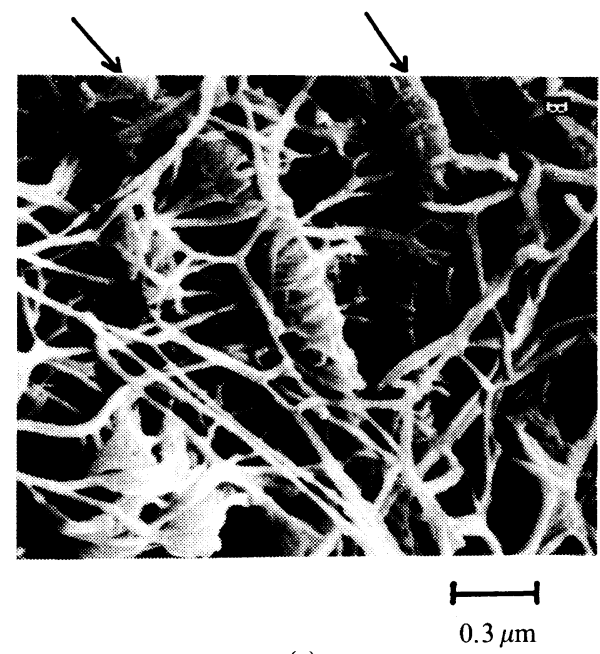

(c)

Figure 1. SEM photograph of a fracture surface for a pressure-crystallized powder sample of UHMW-PE $\left(\bar{M}_{v}\right.$ $2.5 \times 10^{6}$ ). The sample was obtained by crystallization of the original powder sample at the cooling rate of $2 \mathrm{~K} \mathrm{~min}^{-1}$ at $590 \mathrm{MPa}$ from the melt. Arrow shows fibrous band. The direction of the arrow indicates the direction of long axis of the band. 
Figures 1(b) and (c) show the fibrous bands. The direction of the arrow indicates the direction of long axis of the band. The width of the fibrous band is about $0.2 \mu \mathrm{m}$, and the fibrous band is formed by the parallel arrangement of the long fibrils which orient perpendicular to the long axis of the band. The long axis of the fibrous band is directed approximately in the same direction as shown in Figure 1(b).

Electron micrographs of the original powder sample are shown in Figures 2(a), (b), and (c). The sample is composed of an aggregate of many particles (Figure 2(a)) of diameter about $10 \mu \mathrm{m}$. The particle is an aggregate of many small globular particles as shown in Figure 2(b). The diameter of a small globular particle is about $0.5 \mu \mathrm{m}$. The network structure consisting of many fibrils is observed on the surface of the small globular particle in Figure 2(c), and the small globular particles are linked by many other fibrils as indicated by the arrows in Figure 2(c). The diameter of the fibrils is approximately $500 \AA$ which is the same as that of the pressure-crystallized sample as appeared in Figure 1(c). The pressure-crystallized powder sample was obtained by holding the sample in the molten state for about 20 minutes followed by cooling under high pressure. However, the diameter of the fibril in the original powder sample does not change so

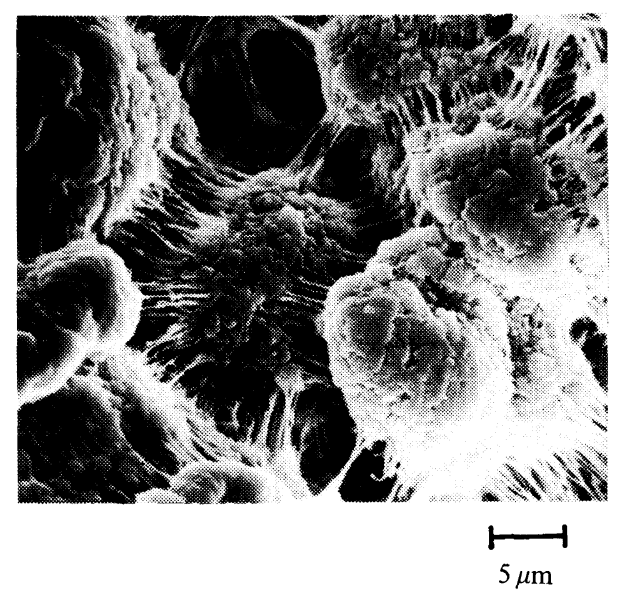

(a) much by HPC.

As mentioned in a previous paper, ${ }^{10}$ high pressure DTA for the powder sample was carried out by coating epoxy resin on it. The melting and crystallization temperatures of this sample obtained by the high pressure DTA were higher than those for bulk sample. In addition, these temperatures increase linearly with increasing pressure. These facts indicate that the applied pressure is transmitted in the powder sample perfectly. The HPC

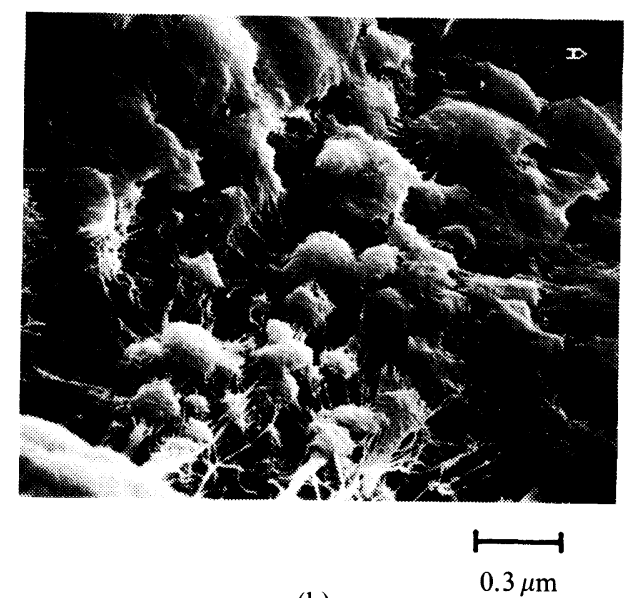

(b)

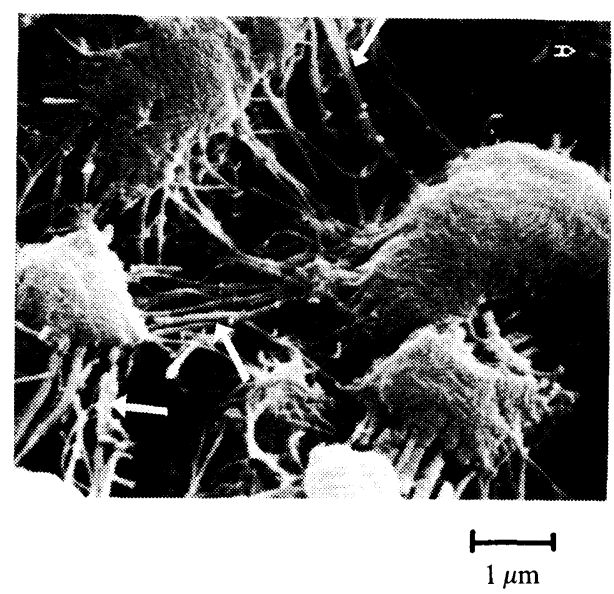

(c)

Figure 2. SEM photograph of an original powder sample of UHMW-PE $\left(\bar{M}_{v} 2.5 \times 10^{6}\right)$. Arrow in (c) indicates fibrils which link small globular particles. 
of the powder sample in the present experiment was also performed by coating epoxy resin on it, so it is reasonable to consider that the HPC was performed under a uniform hydrostatic pressure.

Though the hydrostatic pressure was applied in the powder sample, the pressurecrystallized powder sample is very porous. This fact indicates that coalescence between particles did not occur largely in the HPC process of the powder sample, and that a vast volume expansion occurred in the releasing process of the applied pressure. Actually, the apparent density of the pressure-crystallized powder sample was very low $\left(c a .0 .5 \mathrm{~g} \mathrm{~cm}^{-3}\right)$ at atmospheric pressure.

As shown in Figures 1 and 2, the morphology of the pressure-crystallized powder sample is drastically changed compared with that of the original powder sample. The fibrous band is strikingly different in morphology from that of the band structure of MMW-PE, indicating that the formation mechanism of fibrous band is different from that of the band structure in MMW-PE. ${ }^{15,16}$

Zachariades et al. have studied melting behavior of UHMW-PE at atmospheric pressure. ${ }^{17,18}$ In the studies on UHMW-PE fibers with draw ratio 150 obtained by solid state drawing of a single crystal mat, they observed that the birefringence remained up to $240^{\circ} \mathrm{C}$ when the fibers were heated at a heating rate of $10 \mathrm{~K} \mathrm{~min}^{-1}$ with the ends fixed. They suggested that complete fusion of the crystalline phase occurred when the sample was heated to a temperature $\left(>220^{\circ} \mathrm{C}\right)$ where sufficient disentanglement and chain translation might occur. In the present experiment the sample for the $\mathrm{HPC}$ was heated up to $295^{\circ} \mathrm{C}$ at $590 \mathrm{MPa}$. The temperature is 28 and $40^{\circ} \mathrm{C}$ higher than those of the peak melting temperatures in high pressure DTA for the powder and bulk samples, respectively. Accordingly, it is reasonable to consider that the complete fusion of the fibrils in these samples did not occur easily at $295^{\circ}$ C. As shown in Figures 1 and 2, the shape of the fibril in the original sample remains in the fibrous band due to the difficulty of the complete fusion of the fibrils in the HPC process.

The formation mechanism of fibrous band is assumed as follows. Since the average chain length of this sample is very long ( $c a .20 \mu \mathrm{m})$, many entanglements between molecular chains in the fibril should exist. Accordingly, in spite of the melting of crystallites in the fibrils, the shape of the fibrils changes scarcely even above its melting temperature. As a matter of course, the flexibility of the fibrils increases above its melting temperature. Then parallel arrangement of the fibrils is made by pressure so as to be packed most closely. The parallel arrangement results in the fibrous band in its morphology.

DSC melting curves for the original and pressure-crystallized powder and bulk samples are shown in Figures 3(a) and (b), respectively. The DSC was performed at a heating rate of

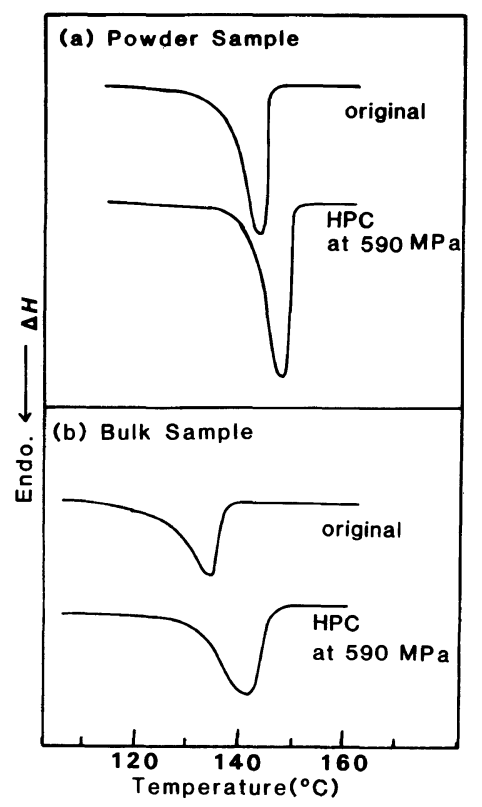

Figure 3. DSC melting curves for the original and pressure-crystallized powder and bulk samples. (a) powder sample, (b) bulk sample. The heating rate was $4 \mathrm{~K} \mathrm{~min}^{-1}$. 
Table I. Melting peak temperatures and heats of fusion for the original and pressure-crystallized powder and bulk samples at atmospheric pressure. $T_{\mathrm{p}}$, peak temperature of DSC curve; $\Delta H$, heat of fusion calculated from the area of DSC curve

\begin{tabular}{cccccc}
\hline & \multicolumn{2}{c}{ Powder } & & \multicolumn{2}{c}{ Bulk } \\
\cline { 2 - 3 } \cline { 5 - 6 } \cline { 5 - 6 } & Original & Pressure-crystallized & & Original & Pressure-crystallized \\
\hline$T_{\mathrm{p} /{ }^{\circ} \mathrm{C}}$ & 143.2 & 147.6 & & 132.8 & 140.2 \\
$\Delta H / \mathrm{cal} \mathrm{g}^{-1}$ & 44 & 54 & & 36 & 48 \\
\hline
\end{tabular}

$4 \mathrm{Kmin}^{-1}$. Melting peak temperatures and heat of fusions calculated from the area of the peak for these samples are listed in Table I. The listed values are averaged ones obtained from several DSC measurements.

The increase of the melting peak temperature of the original powder and bulk samples by the HPC $\left(143.2 \rightarrow 147.6^{\circ} \mathrm{C}, 132.8 \rightarrow 140.2^{\circ} \mathrm{C}\right)$ indicates the increase of lamellar thickness. It has been reported that the crystallinity of the pressure-crystallized MMW-PE is close to unity. ${ }^{1,11}$ The heat of fusion of the equilibrium crystal of $\mathrm{PE}$ is about $70 \mathrm{calg}^{-1} \cdot{ }^{19}$ However, as shown in Table I, the heat of fusion of the pressure-crystallized powder sample is much lower than that of the equilibrium crystal. One reason for the lower heat of fusion of the pressure-crystallized powder sample is supposed to be due to the lower crystallinity of the sample and the increase of defects in the sample caused due to the difficulty in extending the long chain molecule during the crystallization process.

The DSC measurement of the pressurecrystallized UHMW-PE was performed by Hoehn et al. ${ }^{13}$ at a heating rate of $10 \mathrm{~K} \mathrm{~min}^{-1}$ at atmospheric pressure. The peak temperature of the pressure-crystallized powder sample presented by them is slightly higher than the values obtained in the present experiment by the effect of superheating. The heat of fusion of the pressure-crystallized powder sample in our experiment is $54 \mathrm{cal} \mathrm{g}^{-1}$ which is slightly lower than the value obtained by them.
Scanning electron micrographs of a fracture surface for pressure-crystallized bulk sample of UHMW-PE are shown in Figures 4(a) and (b). As shown in the figures, there are three types in the morphology of the fracture surface, i.e., isolated fibril, textile structure and band structure. Arrows A and B in Figure 4(a) indicate the textile structure and the band structure, respectively. The textile structure is formed by the network of the fibrils. The band structure in Figure 4(b) is composed of the parallel arrangement of fibrils as observed in the pressure-crystallized powder sample.

The fibrils of the original UHMW-PE powder were heated above the melting temperature in the preparation process of the bulk sample and its HPC process. That is, the fibrils were held in the molten state for a long time. Therefore, the boundaries of the fibrils in the textile structure and the band structure are not clear. By the coalescence with adjacent fibrils in the molten state the fibrils on the band structure (Figure 4(b)) are apparently wider than those of the pressure-crystallized powder sample (Figure 1(b)). The width of the band in the bulk sample is approximately $0.5 \mu \mathrm{m}$ and is also wider than that of the pressurecrystallized powder sample.

It seems to be commonly accepted that the band structure is formed by the growth of $\mathrm{ECC}$, i.e., ECC grows in the crystallization process. As mentioned above, however, the formation of parallel arrangement of fibrils in UHMW-PE proceeds in the molten state, i.e., the basic structure of the band is formed in the 


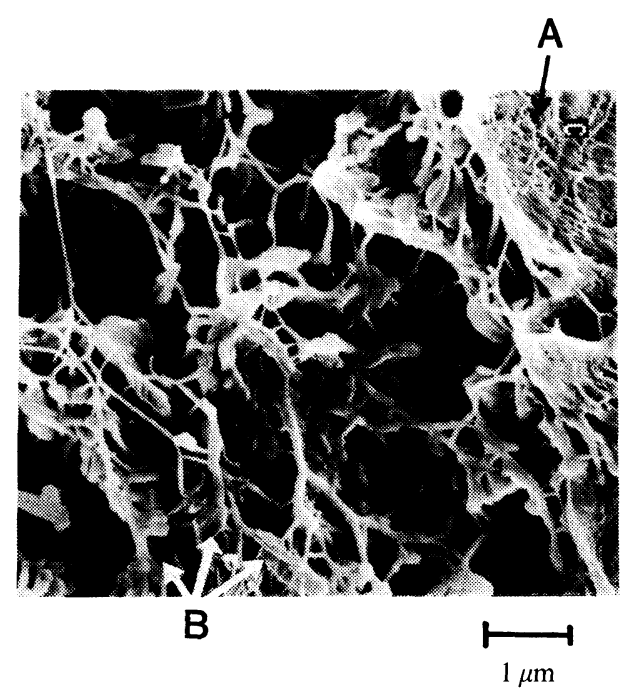

(a)

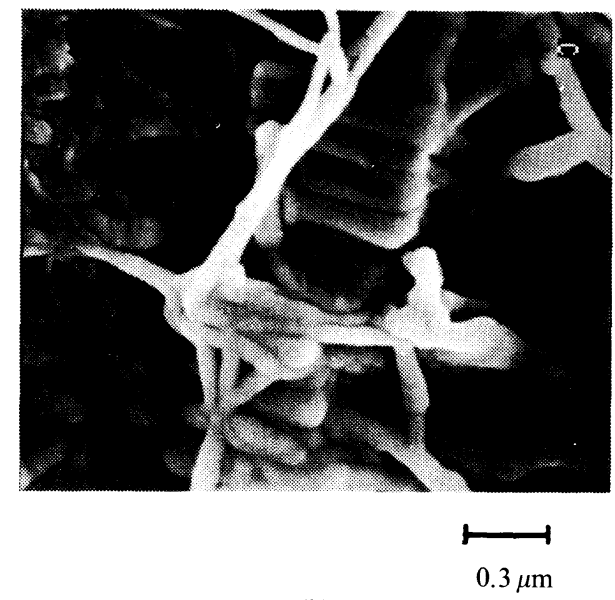

(b)

Figure 4. SEM photograph of a fracture surface for a pressure-crystallized bulk sample of UHMW-PE $\left(\bar{M}_{v}\right.$ $2.5 \times 10^{6}$ ). The sample was obtained by the crystallization of the original powder sample at a cooling rate of $2 \mathrm{~K} \mathrm{~min}^{-1}$ at $590 \mathrm{MPa}$ from the melt. Arrows A and B in (a) indicate the textile structure and the band structure, respectively.

molten state. Therefore the formation of the band in UHMW-PE occurs prior to the growth of ECC independent of the growth of ECC.

As shown in Figure 2(c), the surface of the globular particles of the original powder sam-

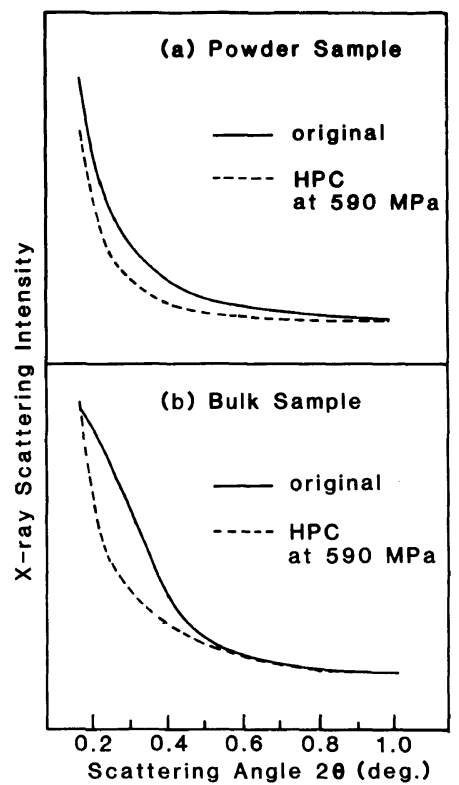

Figure 5. Small angle X-ray scattering intensity profiles for the original and pressure-crystallized samples.

ple is like a textile which consists of network of the fibrils. The pressure-crystallized bulk sample was obtained through the process of the HPC on the kneaded sample; nevertheless, the textile structure of this sample is substantially the same as that of the original powder sample. Accordingly, the textile structure in the pressure-crystallized bulk sample is the survivor of that of the original powder sample.

Figure 5 shows SAXS profiles for the original and pressure-crystallized powder and bulk samples. The measurements were carried out in the scattering angle range from $2 \theta=0.16^{\circ}$ to $1.00^{\circ}$. The SAXS profile for the pressurecrystallized bulk sample is the same as that of the original powder sample, except that the intensity of the former is slightly higher than the latter around $0.4^{\circ}$ in $2 \theta$.

As shown in Figure 5, the scattering intensity around $0.3^{\circ}$ for the original powder and bulk samples decreased on HPC. The angle of $0.3^{\circ}$ corresponds approximately to the long period of $300 \AA$. The lamellar thickness of 
$300 \AA$ corresponds to the melting temperature of $132^{\circ} \mathrm{C}^{19}$ On the other hand, the DSC melting peaks for the original powder and bulk samples shift to higher temperatures by the HPC as shown in Figure 3. The shift of the melting peak indicates the increase of the lamellar thickness of the original powder and bulk samples by the HPC. Accordingly, the decrease of the scattering intensity around the angle of $0.3^{\circ}$ can be explained by the increase of the lamellar thickness.

The DSC melting peak for the original bulk sample is broader than that of the original powder sample. Though the DSC melting peak of the bulk sample shifts to a higher temperature on pressure-crystallization, the breadth of the DSC melting peak does not change. This indicates that in spite of the increase of the lamellar thickness the widely spreaded distribution of the lamellar thickness of the original bulk sample does not change by the HPC. The wide distribution of the lamellar thickness of the original bulk sample is shown by the high $\mathrm{X}$-ray scattering intensity in an angle range below $0.5^{\circ}$ in $2 \theta$ (Figure $5(\mathrm{~b})$ ). For the pressure-crystallized bulk sample, however, the wide distribution of the lamellar thickness suggested by the broad DTA melting peak cannot be confirmed by the present SAXS profile due to the increase of the lamellar thickness beyond the resolution of the experimental condition.

The melting temperature of the pressurecrystallized bulk sample is lower than that of the pressure-crystallized powder sample as shown in Figure 3 and Table I. These facts indicate that the average lamellar thickness of the pressure-crystallized bulk sample is smaller than that of the pressure-crystallized powder sample. In addition, as mentioned above the lamellar thickness in the pressure-crystallized bulk sample is widely spread. From these results, the microstructure of the pressurecrystallized bulk sample is assumed as follows. The fibrils in the textile structure of the pressure-crystallized bulk sample are not straight but winding and bending as shown in Figure 4. The twisting of fibrils should be accompanied by winding and bending. On the other hand, the isolated fibrils and fibrils in the band are comparatively straight, but the quantity of the isolated fibrils and band in the pressure-crystallized bulk sample is small. In the winding, twisting and bending portion, many entanglements of molecular chain might be included, and the entanglement might hinder the growth of crystals. ${ }^{10}$

Hoehn et al. ${ }^{12}$ have suggested "strand-like network superstructure of lamellae" for the morphology of pressure-crystallized UHMWPE etched by nitric acid. Their "lamellar" thickness of UHMW-PE $\left(\bar{M}_{w}=4.6 \times 10^{6}\right)$ sample ranges from 0.75 to $0.18 \mu \mathrm{m}$ which is comparable to the width of fibrous band of the pressure-crystallized powder sample and that of the band of pressure-crystallized bulk sample $(0.2$ and $0.5 \mu \mathrm{m})$. Thus, their "lamellar" likely corresponds to the fibrous band and the band structure in this paper. They have not reported the detailed structure of the "lamellar." As mentioned above, the detailed structure of the fibrous band and the band structure are clarified in the present investigation by the use of high resolution SEM.

Acknowledgments. The authors would like to thank for Hitachi, Ltd. for taking high magnification electron micrographs with field emission type SEM (S-800). We are also indebted to Miss. M. Eguchi and Mr. T. Sonobe for their assistance with the thermal analysis and the SAXS measurement and $\mathrm{Mr}$. T. Itou for his advice on the experiment of electron microscopy.

\section{REFERENCES}

1. B. Wunderlich and T. Arakawa, J. Polym. Sci., A-2, 7, 3697 (1964).

2. P. H. Geil, F. R. Anderson, B. Wunderlich, and T. Arakawa, J. Polym. Sci., A-2, 7, 3707 (1964).

3. B. Wunderlich, "Macromolecular Physics," Vol. 1, 
Academic Press, New York, N. Y., 1973.

4. B. Wunderlich, "Macromolecular Physics," Vol. 2, Academic Press, New York, N. Y., 1976.

5. B. Wunderlich, "Macromolecular Physics," Vol. 3, Academic Press, New York, N. Y., 1980.

6. D. C. Bassett, Polymer, 17, 460 (1976).

7. D. C. Bassett, "Developments in Crystalline Polymers," Vol. 1, Applied Science, London, 1982, Chapter 3 .

8. S. K. Bhateja and K. D. Pae, J. Macromol. Sci., Rev. Macromol. Chem., C13, 77 (1975).

9. U. Leute and W. Dollhopf, Colloid Polym. Sci., 258, 353 (1980).

10. M. Yasuniwa, K. Haraguchi, C. Nakafuku, and S. Hirakawa, Polym. J., 17, 1209 (1985).

11. M. Yasuniwa, C. Nakafuku, and T. Takemura, Polym. J., 4, 526 (1973).
12. H. H. Hoehn, R. C. Ferguson, and R. R. Hebert, Polym. Eng. Sci., 18, 457 (1978).

13. R. C. Ferguson and H. H. Hoehn, Polym. Eng. Sci., 18, 466 (1978).

14. N. Hiramatsu and S. Hirakawa, Polym. J., 12, 105 (1980).

15. T. Asahi, Y. Miyamoto, H. Miyaji, and K. Asai, Polymer, 23, 773 (1982).

16. S. Miyashita, T. Asahi, H. Miyaji, and K. Asai, Polymer, 26, 1791 (1985).

17. A. E. Zachariades and J. A. Logan, J. Polym. Sci., Polym. Phys. Ed., 21, 821 (1983).

18. A. E. Zachariades and T. Kanamoto, Polym. Eng. Sci., 26, 658 (1986).

19. B. Wunderlich, "Macromolecular Physics," Vol. 3, Academic Press, New York, N. Y., 1980, Chapter 8. 